University of Wollongong

Research Online

Australian Institute for Innovative Materials -

Papers

Australian Institute for Innovative Materials

$1-1-2016$

\title{
Strong affinity of polysulfide intermediates to multi-functional binder for practical application in lithium-sulfur batteries
}

\author{
Hongqiang Wang \\ University of Wollongong, hw571@uowmail.edu.au \\ Vitor Sencadas \\ University of Wollongong, victors@uow.edu.au \\ Guoping Gao \\ Queensland University of Technology \\ Hong Gao \\ University of Wollongong, hg173@uowmail.edu.au
}

Aijun Du

Queensland University of Technology

See next page for additional authors

Follow this and additional works at: https://ro.uow.edu.au/aiimpapers

Part of the Engineering Commons, and the Physical Sciences and Mathematics Commons

Research Online is the open access institutional repository for the University of Wollongong. For further information contact the UOW Library: research-pubs@uow.edu.au 


\title{
Strong affinity of polysulfide intermediates to multi-functional binder for practical application in lithium-sulfur batteries
}

\author{
Abstract \\ Binder, one of the most important battery components, plays a critical role in lithium-sulfur batteries. \\ Poly(vinylidene difluoride) (PVDF), a commonly used binder in lithium-sulfur batteries, does not have a \\ strong affinity to the intermediate polysulfides, however, leading to fast capacity fading with \\ electrochemical cycling. Herein, copolymers of vinylidene difluoride with other monomers are used as \\ multi-functional binders to enhance the electrochemical performance of lithium-sulfur batteries. \\ Compared to the PVDF, the copolymer, poly(vinylidene difluoride-trifluoroethylene) (P(VDF-TRFE)) binder \\ exhibits higher adhesion strength, less porosity, and stronger chemical interaction with polysulfides, \\ which helps to keep the polysulfides within the cathode region, thereby improving the electrochemical \\ performance of the lithium-sulfur battery. As a result, sulfur electrode with $\mathrm{P}$ (VDF-TRFE) binder delivered a \\ high capacity of $801 \mathrm{~mA} \mathrm{~h} \mathrm{g-1}$ at $0.2 \mathrm{C}$ after 100 cycles, which is nearly $80 \%$ higher capacity than the \\ corresponding sulfur cathode with PVDF binder. \\ Disciplines \\ Engineering | Physical Sciences and Mathematics

\section{Publication Details} \\ Wang, H., Sencadas, V., Gao, G., Gao, H., Du, A., Liu, H. \& Guo, Z. (2016). Strong affinity of polysulfide \\ intermediates to multi-functional binder for practical application in lithium-sulfur batteries. Nano Energy, \\ $26722-728$.
}

\section{Authors}

Hongqiang Wang, Vitor Sencadas, Guoping Gao, Hong Gao, Aijun Du, Hua-Kun Liu, and Zaiping Guo 


\title{
Strong Affinity of Polysulfide Intermediates to Multi-Functional Binder for Practical Application in Lithium-Sulfur Batteries
}

\author{
Hongqiang Wang ${ }^{\mathrm{a}}$, Vitor Sencadas ${ }^{\mathrm{b}}$, Guoping $\mathrm{Gao}^{\mathrm{c}}, \mathrm{Hong} \mathrm{Gao}^{\mathrm{a}}$, Aijun $\mathrm{Du}^{\mathrm{c}}$, Huakun Liu ${ }^{\mathrm{a}}$, \\ Zaiping $\mathrm{Guo}^{\mathrm{a}, \mathrm{b}_{*}}$
}

\footnotetext{
${ }^{a}$ Institute for Superconducting \& Electronic Materials, University of Wollongong, NSW 2522, Australia Email: zguo@uow.edu.au

${ }^{\mathrm{b}}$ School of Mechanical, Materials \& Mechatronics Engineering, University of Wollongong, NSW 2500, Australia.

${ }^{c}$ School of Chemistry, Physics and Mechanical Engineering, Queensland University of Technology, QLD 4001, Australia.
}

Abstract: Binder, one of the most important battery components, plays a critical role in lithium-sulfur batteries. Poly(vinylidene difluoride) (PVDF), a commonly used binder in lithium-sulfur batteries, does not have a strong affinity to the intermediate polysulfides, however, leading to fast capacity fading with electrochemical cycling. Herein, copolymers of vinylidene difluoride with other monomers are used as multi-functional binders to enhance the electrochemical performance of lithium-sulfur batteries. Compared to the PVDF, the copolymer, poly(vinylidene difluoride-trifluoroethylene) (P(VDF-TRFE)) binder exhibits higher adhesion strength, less porosity, and stronger chemical interaction with polysulfides, which helps to keep the polysulfides within the cathode region, thereby improving the electrochemical performance of the lithium-sulfur battery. As a result, sulfur electrode with $\mathrm{P}\left(\right.$ VDF-TRFE) binder delivered a high capacity of $801 \mathrm{mAh} \mathrm{g}^{-1}$ at $0.2 \mathrm{C}$ after 100 cycles, which is nearly $80 \%$ higher capacity than the corresponding sulfur cathode with PVDF binder. KEYWORDS: lithium-sulfur batteries; copolymer binder; chemical affinity; polysulfide intermediates; density functional theory (DFT) calculations 


\section{Introduction}

Lithium-sulfur (Li-S) battery is believed to be one the most promising candidates among the proposed rechargeable battery systems because of its high theoretical specific capacity (1675 $\mathrm{mAh} \mathrm{g}^{-1}$ ), inexpensive cost, and environmentally friendliness [1-5]. Nevertheless, the sulfur cathodes face several persistent problems, such as the poor conductivity of sulfur, high solubility of polysulfides in the electrolyte, and large volume changes during the cycling process. These result in low sulfur utilization, low coulombic efficiencies, and rapid capacity fading of Li-S batteries [6-12]. Many strategies have been applied to Li-S batteries to address these scientific issues in the past few years, mainly focusing on the construction of nanostructured sulfur cathode [13-18] and functionalization of the separator [19-24]. Although these strategies could greatly enhanced the performance of Li-S batteries, the complicated synthesis procedures and the high cost of these matrix materials make them difficult to use in practical applications.

To date, there has been little research focus on industrially viable solutions to the problems of Li-S batteries. The binder, as one of the most important battery components, plays an important role in the electrode fabrication process, and it also has a strong influence on the electrochemical performance of Li-S batteries. Therefore, if the most serious problems of these batteries could be addressed through the choice of a suitable binder, the electrode preparation process and the whole battery set-up could still be kept simple, which would greatly promote the practical application of Li-S batteries. A suitable binder in the Li-S system should have strong adhesion to ensure good contact between the sulfur-based nanomaterial and the conductive carbon and current collector, as well as good mechanical stability against volumetric expansion of sulfur, and it also could help to control polysulfide dissolution and diffusion. 
Poly(vinylidene difluoride) (PVDF) is a semicrystalline polymer known for its polymorphism that is also widely used as a binder in Li-S batteries. Although it has relatively strong adhesion and can link the sulfur-based active material to the current collector, its affinity to polysulfide is weak, resulting in poor cycling stability. Compared to PVDF homopolymer, copolymers of vinylidene difluoride (VDF) with different monomers have different properties in terms of adhesive strength, porosity, and polarity $[25,26]$, which are important factors that influence the electrochemical performance of Li-S batteries. Recently, it was reported that polar materials, such as $\mathrm{TiO}_{2}, \mathrm{Ti}_{4} \mathrm{O}_{7}$, and $\mathrm{MnO}_{2}$, are thought to bind with lithium polysulfides in form of chemical bonds that would keep them within the cathode region, thus improving the cycling stability of Li-S batteries [27-31]. Inspired by this, herein, we have used copolymers of VDF with other monomers as binders to improve the electrochemical performance of Li-S batteries. Compared to the PVDF homopolymer, our copolymers demonstrate higher adhesion strength, enhanced conductivity, less porosity, higher polarity, and strong affinity with polysulfides, which contribute to the good performance of Li-S batteries.

\section{Experimental Section}

\section{Preparation of ordered mesoporous carbon}

An ordered mesoporous carbon was prepared through the method reported by Zhao et al [32]. Then, the silica was removed by putting the mesoporous carbon-silica composite into $2 \mathrm{M}$ $\mathrm{NaOH}$ solution $\left(100^{\circ} \mathrm{C}\right)$ for $2 \mathrm{~h}$.

\section{Fabrication of sulfur cathodes}

The sulfur (70 wt $\%)$ and as-prepared mesoporous carbon (30\%) were mixed and then heated to $160{ }^{\circ} \mathrm{C}$ in a sealed tube for $20 \mathrm{~h}$. The carbon-sulfur composite ( $70 \%$ by weight) was mixed with Super-P (10\%) and binder (PVDF from Solvay, poly(vinylidene difluoridetrifluoroethylene) $[\mathrm{P}(\mathrm{VDF}-\mathrm{TRFE})]$ and poly(vinylidene difluoride-co-chlorotrifluoroethylene) 
[(P(VDF-co-CTFE)] from Piezotech) (20\%) in N-methyl-2-pyrrolidone (NMP), respectively, to form the slurry. After that, the slurry was coated on aluminum foil to prepare sulfur electrodes and then dried at $50{ }^{\circ} \mathrm{C}$ for $24 \mathrm{~h}$.

\section{Characterization}

The sulfur content in the composite was measured by a thermogravimetric analysis (TGA, METTLER TOLEDO, Switzerland) instrument from room temperature to $500{ }^{\circ} \mathrm{C}$ at a heating rate of $10{ }^{\circ} \mathrm{C}$ per min in argon atmosphere. The morphologies and compositions of the samples were investigated by a scanning electron microscope (SEM; JEOL JSM-7500FA) equipped with energy-dispersive X-ray (EDX) spectroscopy, and a transmission electron microscope (TEM; JEOL 2011). The adhesive strength of the polymeric binder with respect to the aluminum foil was evaluated using universal testing machine from Tensile \& Compression Testers (INSTRON 5943). The aluminum strips were cleaned with ethanol, and then the binder solutions were spread on the strips, with a coated area $1 \mathrm{~cm}$ in length, $1 \mathrm{~cm}$ in width, and $0.2 \mathrm{~mm}$ in thickness. Subsequently, two coated areas were overlapped and bonded together. The strips were dried under vacuum at $50{ }^{\circ} \mathrm{C}$ for $24 \mathrm{~h}$ before measurements.

\section{Electrochemical measurements}

The CR2032 coin cells were assembled in a glovebox filled with argon. The electrolyte was 1 M LiTFSI in a mixture of 1,3-dioxolane (DOL) and dimethoxyethane (DME) (1:1 by volume) containing $\mathrm{LiNO}_{3}(2 \mathrm{wt} \%)$. The electrochemical performance of the coin cells was tested within a voltage window of 1.8-2.6 V using a LAND CT2001A battery test system.

\section{Results and discussion}

Figure 1a shows the molecular structure of PVDF and its copolymers with other monomers. PVDF has two fluorine atoms in the monomer unit, while the monomer of P(VDF-TRFE) has five fluorine atoms and one hydrogen. In the case of the P(VDF-co-CTFE), the hydrogen in the trifluorethylene is replaced by a chloride atom. The modification observed in the 
monomer unit enhances the molecular polarity of the VDF based copolymers. Polar materials are thought to have a strong affinity to lithium polysulfides, effectively keeping them within the cathode region and thus improving the electrochemical stability of the Li-S battery [27, 30]. Therefore, we have used ab initio simulations performed in the framework of density functional theory to clearly confirm the interaction between $\mathrm{Li}_{2} \mathrm{~S}$ and the polymer binders (Figure $1 \mathrm{~b}$ ). The strongest interaction with $\mathrm{Li}_{2} \mathrm{~S}$ can be observed in the case where it bonds with the $-\mathrm{F}$ group in P(VDF-TRFE) binder, with binding energy of $1.05 \mathrm{eV}$ and bond length of $1.972 \AA$. In contrast, lower binding energy of $0.78 \mathrm{eV}$ was found in the case where $\mathrm{Li}_{2} \mathrm{~S}$ bonds with the $-\mathrm{F}$ group in the conventional PVDF binder. Furthermore, P(VDF-TRFE) binder exhibits a high binding energy of $0.876 \mathrm{eV}$ with $\mathrm{Li}-\mathrm{S} \cdot$ species (referring to the relevant end groups in the general class of lithium polysulfides, $\mathrm{Li}-\mathrm{S}-\mathrm{S}_{\mathrm{n}-2}-\mathrm{S}-\mathrm{Li} ; \mathrm{Li}_{2} \mathrm{~S}_{\mathrm{n}}$ for short, $4 \leq \mathrm{n} \leq 8)$ [33], as shown in Figure 1c, which is higher than the binding energy $(0.75 \mathrm{eV})$ between lithium polysulfides and PVDF binder. The $a b$ initio simulation results demonstrate that the $-\mathrm{F}$ group in the $\mathrm{P}(\mathrm{VDF}-\mathrm{TRFE})$ binder possesses a stronger affinity to $\mathrm{Li}_{2} \mathrm{~S}$ and lithium polysulfides than it does in the PVDF binder, which will play a critical role in terms of maintaining stable electrochemical performance.

The sulfur cathodes were prepared by using mesoporous carbon-sulfur composite (Supporting Information Figures S1 and S2) as the active material, Super P as conductive additive, and PVDF and its copolymers (P(VDF-co-CTFE) and P(VDF-TRFE)) as binders (with the resultant electrodes denoted as S@PVDF, S@P(VDF-co-CTFE), and S@P(VDF-TRFE)). The cycling performances of the S@PVDF, S@P(VDF-co-CTFE), and S@P(VDF-TRFE) electrodes tested at current density of $0.2 \mathrm{C}$ are shown in Figure 2a. The S@P(VDF-coCTFE) and S@P(VDF-TRFE) electrodes delivered more stable capacity during cycling than the S@PVDF electrode. After 100 cycles, high reversible discharge capacities of 650 and 801 $\mathrm{mAh} \mathrm{g}^{-1}$ were obtained for the S@P(VDF-co-CTFE) and S@P(VDF-TRFE) electrodes, 
respectively. In contrast, it was observed that the discharge capacity of the S@PVDF

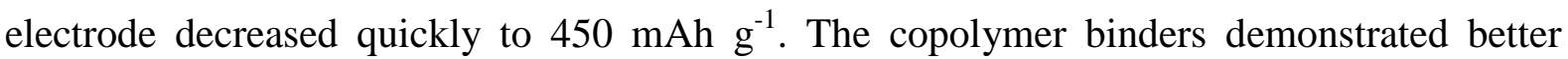
capacity retention than the homopolymer PVDF one. Furthermore, the S@P(VDF-TRFE) electrode delivered higher initial discharge capacity and coulombic efficiency (Figure S3) compared to the S@PVDF electrode, demonstrating enhanced sulfur utilization. The discharge curves of the S@PVDF, S@P(VDF-co-CTFE), and S@P(VDF-TRFE) electrodes for selected cycles are shown in Figure $2 b-d$, in which two typical plateaus representing the sulfur cathode at around 2.1 and $2.3 \mathrm{~V}$ can be observed from the discharge curves. It was reported that the higher discharge plateau at $2.3 \mathrm{~V}$ is attributed to the formation of soluble high-order polysulfides [21]. After 100 cycles, the retention rate of the upper plateau discharge capacity is $72.9 \%$ and $56.1 \%$ for the S@P(VDF-TRFE) and S@P(VDF-co-CTFE) electrodes, respectively, while only $41.8 \%$ is retained for the S@PVDF electrode (Figure S4). Therefore, the high retention rate of the upper plateau discharge capacity indicates that the $\mathrm{P}(\mathrm{VDF}-\mathrm{TRFE})$ binder has strong polysulfide-trapping capability, which can keep the polysulfides within the cathode region due to its higher affinity with them, resulting in good cycling stability.

Figure 2e displays the rate performances of the S@PVDF, S@P(VDF-co-CTFE), and S@P(VDF-TRFE) electrodes. Even at current density of 5 C, a satisfactory capacity of nearly $450 \mathrm{mAh} \mathrm{g}^{-1}$ can be obtained for the S@P(VDF-TRFE) electrode, which is much higher than that of the S@PVDF, confirming the excellent rate capability due to the strong interaction between the P(VDF-TRFE) binder and the polysulfides, and the improved reaction kinetics. It is notable that the $\mathrm{S} @ \mathrm{P}(\mathrm{VDF}-\mathrm{TRFE})$ and $\mathrm{S} @ \mathrm{P}(\mathrm{VDF}-\mathrm{co}-\mathrm{CTFE})$ electrodes show lower resistance and higher lithium ion conductivity, as confirmed by the diffusion coefficients $\left(4.03 \times 10^{-11} \mathrm{~cm}^{2} \mathrm{~s}^{-1}, 2.29 \times 10^{-10} \mathrm{~cm}^{2} \mathrm{~s}^{-1}\right.$, and $3.90 \times 10^{-10} \mathrm{~cm}^{2} \mathrm{~s}^{-1}$ for the S@PVDF, $\mathrm{S} @ \mathrm{P}(\mathrm{VDF}-\mathrm{co}-\mathrm{CTFE})$, and S@P(VDF-TRFE) electrodes, respectively) (Figure S5), which 
also contributes to the rate capability. The long-term cycling performances of sulfur electrodes with the different binders at $0.5 \mathrm{C}$ are presented in Figure $2 \mathrm{f}$. After 300 cycles, the $\mathrm{S} @ \mathrm{P}(\mathrm{VDF}-\mathrm{TRFE})$ electrode can still deliver $540 \mathrm{mAh} \mathrm{g}^{-1}$, corresponding to $59.2 \%$ capacity retention of its initial capacity and a small capacity fading of only $0.136 \%$ per cycle. The S@PVDF electrode, however, only delivers capacity of $180 \mathrm{mAh} \mathrm{g}^{-1}$. Here, we believe that the enhanced electrochemical properties should be attributed to the lower porosity, higher conductivity, and stronger polysulfide-trapping capability of P(VDF-TRFE) binder, which can keep polysulfides within the cathode region. It was reported that when PVDF crystallization from a solvent solution occurs at temperatures below $60^{\circ} \mathrm{C}$, all of the material crystallizes in the polar trans planar "zigzag" $\beta$-phase, but it also presents higher porosity $[34,35]$, which not only affects the mechanical properties of the electrode, but also the overall conductivity of the electrode. In contrast, when P(VDF-TRFE) copolymer crystallizes from a solvent solution at $50^{\circ} \mathrm{C}$, it was reported that an homogeneous and non-porous film is obtained [36], leading to good mechanical performance. Moreover, the lack of porosity demonstrated by P(VDF-TRFE) probably lead to enhanced wrapping of the sulfur and active carbon particles, improving the adhesion and surface contact between the metal contact and the active materials, which increases the overall conductivity of the electrode.

The adhesive property of the binders was therefore investigated. The lap shear strength curves of the PVDF, P(VDF-co-CTFE), and P(VDF-TRFE) binders are presented in Figure S6. The adhesion strength of the $\mathrm{P}(\mathrm{VDF}-\mathrm{co}-\mathrm{CTFE})$ and $\mathrm{P}(\mathrm{VDF}-\mathrm{TRFE})$ binders is higher than that of the PVDF binder, which can help to buffer the volume changes during the cycling process, preventing active material exfoliation and maintaining the integrity of the electrode, especially under the conditions of long-term cycling. In order to prove the strong chemical interaction between the P(VDF-TRFE) binder and the polysulfides, the cycled S@PVDF and S@P(VDF-TRFE) electrodes were examined by ultraviolet-visible (UV-vis) absorption 
spectroscopy (Figure 3a,b). For the S@PVDF electrode, the large boost in the peak around $280 \mathrm{~nm}$ referring to $\mathrm{S}_{8}{ }^{2-} / \mathrm{S}_{6}{ }^{2-}$ species demonstrates that significant amounts of polysulfides have detached from the sulfur cathode and dissolved into the electrolyte [37-38]. In contrast, the similar intensities of the peaks for the $\mathrm{S} @ \mathrm{P}(\mathrm{VDF}-\mathrm{TRFE})$ electrodes at different cycles indicate that there is a similar content of polysulfides in the electrolyte, proving the effective chemical interaction between P(VDF-TRFE) binder and polysulfides, i.e. the strong affinity of polysulfides to P(VDF-TRFE) binder helps to trap polysulfides within the sulfur cathode. In addition, photographs of separators after 100 cycles are displayed in Figure S7. The yellow color on the separator corresponding to the S@PVDF electrode indicates that the polysulfides were diffusing from the cathode and some active materials had exfoliated from the electrode and been stuck on the separator. The color on the separators corresponding to the S@P(VDFco-CTFE), and S@P(VDF-TRFE) electrodes is light yellow, without any active materials stuck on the separator, supporting the viewpoint discussed above: that there is strong cohesiveness and chemical interaction between the polysulfides and the P(VDF-TRFE) binder.

The cycled electrodes were further investigated by SEM and EDX spectroscopy (Figure 3ch). Before cycling, there were no differences between the sulfur electrodes with different binders, and active sulfur composite and Super P carbon can be clearly observed in each electrode. Large particle aggregation can be clearly observed, however, in the S@PVDF electrode after 50 cycles, and the EDX results revealed that the aggregated particles were rich in sulfur (Figure 3i, $\mathrm{j}$ and Table S1). Due to the poor chemical interaction between the polysulfides and the PVDF, a certain amount of sulfur could escape from the carbon matrix and aggregate on the surface of the electrode during the charge-discharge process. It has been reported that aggregation is a typical phenomenon, leading to serious disconnection between the sulfur active material and the conductive carbon, thus degrading the performance of the 
sulfur cathode [39]. No obvious changes can be observed in the S@P(VDF-co-CTFE) and S@P(VDF-TRFE) electrodes, however, and the overall morphology and structure were well preserved, demonstrating that the $\mathrm{P}(\mathrm{VDF}-\mathrm{co}-\mathrm{CTFE})$ and $\mathrm{P}(\mathrm{VDF}-\mathrm{TRFE})$ binders have strong chemical interactions with polysulfides, trapping them within the cathode region and preventing the sulfur from aggregating during the cycling process, leading to the enhanced electrochemical performance of these sulfur electrodes.

\section{Conclusions}

In summary, copolymers of VDF with other monomers are introduced as novel binders to be used in sulfur electrodes for lithium-sulfur batteries. The P(VDF-TRFE) binder not only possesses a higher adhesive mechanical strength compared to the normal PVDF binder, but also demonstrates strong chemical interaction with polysulfides due to its high molecular polarity, as demonstrated in ab initio simulations, thus acting as an inhibitor to restrain the dissolution and diffusion of the polysulfides. The resulting S@P(VDF-TRFE) electrode delivers capacity of $801 \mathrm{mAh} \mathrm{g}^{-1}$ at $0.2 \mathrm{C}$ after 100 cycles, while the corresponding electrode with PVDF only retains a capacity of $450 \mathrm{mAh} \mathrm{g}^{-1}$.

\section{Acknowledgments}

This work was supported the Australian Research Council (ARC) through an ARC Discovery project (DP1094261) and ARC-LIEF Grant (LE0882613). The authors also would like to thank Dr Tania Silver for critical reading of the manuscript.

\section{References:}

[1] P.G. Bruce, S.A. Freunberger, L.J. Hardwick, J.M. Tarascon, Nat. Mater. 11 (2012) 19-29.

[2] J. Shim, K.A. Striebel, E.J. Cairns, J. Electrochem. Soc. 149 (2002) A1321-A1325.

[3] P. Novak, K. Muller, KSV. Santhanam, O. Haas, Chem. Rev. 97 (1997) 207-281. 
[4] Y. Yang, G.Y. Zheng, S. Misra, J. Nelson, M.F. Toney, Y. Cui, J. Am. Chem. Soc. 134 (2012) 15387-15394.

[5] L.X. Yuan, H.P. Yuan, X.P. Qiu, L.Q. Chen, W.T. Zhu, J. Power Sources 189 (2009) $1141-1146$

[6] J.T. Zhang, H. Hu, Z. Li, X.W. Lou, Angew. Chem. Int. Ed. 55 (2016) 3982-3986.

[7] N. Jayaprakash, J. Shen, S.S. Moganty, A. Corona, L.A. Archer, Angew. Chem. Int. Ed. 50 (2011) 5904-5908.

[8] H.L. Wang, Y. Yang, Y.Y. Liang, J.T. Robinson, Y.G. Li, A. Jackson, Y. Cui, H.J. Dai, Nano Lett. 11(2011) 2644-2647.

[9] Y.V. Mikhaylik, J.R. Akridge, J. Electrochem. Soc. 151 (2004) A1969-1976.

[10] W. Ahn, K.B. Kim, K.N. Jung, K.H. Shin, C.S. Jin, J. Power Sources 202 (2012) 394399.

[11] C.F. Zhang, H.B. Wu, C. Yuan, Z.P. Guo, X.W. Lou, Angew. Chem. Int. Ed. 51 (2012) 9592-9595.

[12] B.L. Ellis, K.T. Lee, L.F. Nazar, Chem. Mater. 22 (2010) 691-714.

[13] L.H. Yu, N. Brun, K. Sakaushi, J. Eckert, M.M. Titirici, Carbon 61 (2013) 245-253.

[14] M.S. Park, B.O. Jeong, T.J. Kim, S. Kim, K.Y. Kim, J.S. Yu, Y. Jung, Y.J. Kim, Carbon 68 (2014) 265-272.

[15] H.Q. Wang, W.C. Zhang, H.K. Liu, Z.P. Guo, Angew. Chem. Int. Ed. 55 (2016) 39923996.

[16] J.L. Wang, Y.S. He, J. Yang, Adv. Mater. 27 (2015) 569-575. 
[17] H.Q. Wang, C.F. Zhang, Z.X. Chen, H.K. Liu, Z.P. Guo, Carbon 81 (2015) 782-787.

[18] Z. Li, J.T. Zhang, Y.M. Chen, J. Li, X.W. Lou, Nat. Commun. 6 (2015) 8850.

[19] S.H. Chung, A. Manthiram, Adv. Funct. Mater. 24 (2014) 5299-5306.

[20] J. Balach, T. Jaumann, M. Klose, S. Oswald, J. Eckert, Adv. Funct. Mater. 25 (2015) $5285-5291$.

[21] S.H. Chung, A. Manthiram, Adv. Mater. 26 (2014) 7352-7357.

[22] H.J. Peng, D.W. Wang, J.Q. Huang, X.B. Cheng, Z. Yuan, F. Wei, Q. Zhang, Adv. Sci. $3(2016) 1500268$.

[23] J.Q. Huang, Q. Zhang, H.J. Peng, X.Y. Liu, W.Z. Qian, F. Wei, Energy Environ. Sci. 7 (2014) 347-353.

[24] Z.Y. Zhang, Y.Q. Lai, Z.A. Zhang, K. Zhang, J. Li, Electrochim. Acta 129 (2014) 55-61.

[25] H.S. Nalwa, Ferroelectric Polymers: Chemistry: Physics, and Applications, 1995, pp. 183-227.

[26] W. Bao, Z. Zhang, Y. Gan, X. Wang, J. Lia, J. Energy Chem. 22 (2013) 790-794.

[27] Q. Zhang, Y. Wang, Z.W. Seh, Z. Fu, R. Zhang, Y. Cui, Nano Lett. 15(2015) 3780-3786.

[28] X. Tao, J. Wang, Z. Ying, Q. Cai, G. Zheng, Y. Gan, H. Huang, Y. Xia, C. Liang, W. Zhang, Y. Cui, Nano Lett. 14 (2014) 5288-5294.

[29] Z. Li, J.T. Zhang, X.W. Lou, Angew. Chem. Int. Ed. 54 (2015) 12886-12890.

[30] Q. Pang, D. Kundu, M. Cuisinier, L.F. Nazar, Nat. Commun. 5 (2014) 4759. 
[31] Z. Liang, G. Zheng, W. Li, Z.W. Seh, H. Yao, K. Yan, D. Kong, Y. Cui, ACS Nano 8 (2014) 5249-5256.

[32] C.F. Xue, B. Tu, D.Y. Zhao, Adv. Funct. Mater. 18 (2008) 3914-3921.

[33] W.Y. Li, Q.F. Zhang, G.Y. Zheng, Z.W. Seh, H.B. Yao, Y. Cui, Nano Lett. 13 (2013) $5534-5540$.

[34] V. Sencadas, R. Gregorio Filho, S. Lanceros-Mendez, J. Non-Cryst. Solids 352 (2006) 2226-2229.

[35] R.E. Sousa, J.C. Ferreira, C.M. Costa, A.V. Machado, M.M. Silva, S. Lanceros-Mendez, J. Polym. Sci. Pol. Phys. 53 (2015) 761-773.

[36] A. Ferreira, J. Silva, V. Sencadas, J.L.G. Ribelles, S. Lanceros-Mendez, Macromol. Mater. Eng. 295 (2010) 523-528.

[37] Y.J. Li, H. Zhan, S.Q. Liu, K.L. Huang, Y.H. Zhou, J. Power Sources 195 (2010) 29452949.

[38] Z.B. Xiao, Z. Yang, L. Wang, H.G. Nie, M. Zhong, Q.Q. Lai, X.J. Xu, L.J. Zhang, S.M. Huang, Adv. Mater. 27 (2015) 2891-2898.

[39] J.L. Wang, Z.D. Yao, C.W. Monroe, J. Yang, Y.N. Nuli, Adv. Funct. Mater. 23 (2013) 1194-1201. 


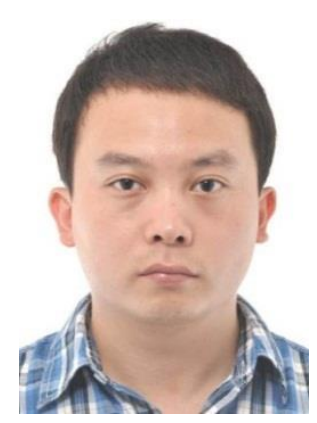

Hongqiang Wang is currently a Ph.D. candidate at Institute for Superconducting and Electronic Materials (ISEM), University of Wollongong, Australia. His research topics focus on smart design and synthesis of nanostructured sulfur cathodes for high-performance lithiumsulfur batteries.

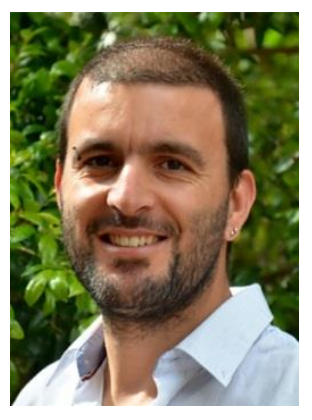

Vitor Sencadas received his Ph.D. degree in Physics at the University of Minho, Portugal, in 2009. He was a research fellow at Centre of Physics of University of Minho and an invited lecture at Polytechnic Institute of Cávado and Ave. Since 2015, he has joined University of Wollongong as a lecture and is a member of the Engineering Materials Strength, leading the smart macromolecules research group and associate investigator of the ARC Centre of Excellence for Electromaterials (ACES, Australia). His work is focused on polymer based smart materials for sensors and actuators, energy storage, biomedical and environmental applications.

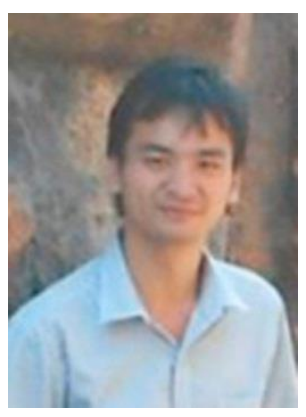

Guoping Gao is currently a Ph.D. candidate at Queensland university of technology, Australia. His research Interests focus on engineering novel 2D materials based catalyst for clean energy conversion reactions, including water splitting and $\mathrm{CO}_{2}$ reduction, based on density function theory (DFT) calculations. 


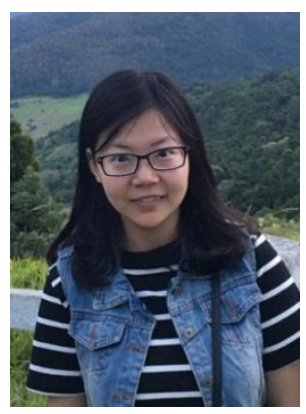

Hong Gao is a Ph.D. student at Institute for Superconducting and Electronic Materials (ISEM), University of Wollongong, Australia. Her current interest is to optimize the sodium-storage properties of promising electrode materials for sodium-ion batteries.

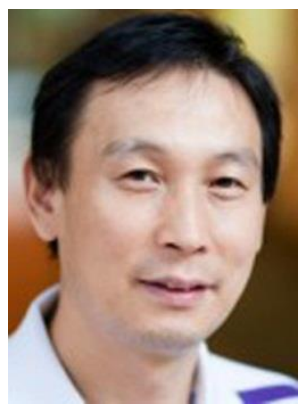

Aijun Du is currently an associate Professor in School of Chemistry, Physics and Mechanical Engineering at Queensland University of Technology (QUT), Australia. He received his Ph.D. degree from Fudan University of China in 2002. Before joining QUT in 2013, he worked at the Australian Institute for Bioengineering and Nanotechnology, the University of Queensland. His research lies at the interface of physics, chemistry and engineering, focusing on developing innovative materials for clean energy, electronics and environmental science through advanced theoretical modeling.

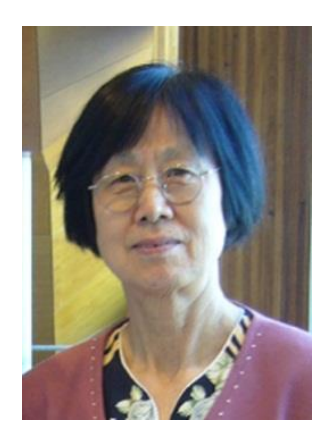

Huakun Liu is a Distinguished Professor and a co-ordinator of energy materials research program of the Institute for Superconducting and Electronic Materials (ISEM) at Australian Institute of Innovative Materials, University of Wollongong. She was elected as a Fellow of the Australian Academy of Technological Science and Engineering in 2013. She has supervised 61 Ph.D. students to completion and 30 postdoctoral and visiting fellows. Her research has been focused on clean energy materials, the materials science and engineering, and electrochemistry and applications. 


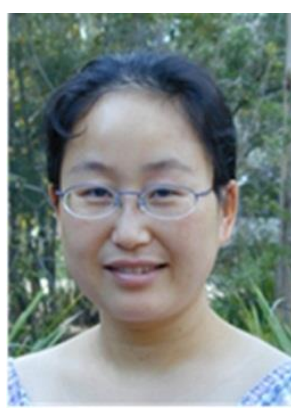

Zaiping Guo obtained her B.S. from the Department of Chemistry, Xinjiang University in 1993 and her Ph.D. from University of Wollongong in 2003. After her postdoctoral research with Prof. Huakun Liu at the Institute for Superconducting and Electronic Materials (ISEM), University of Wollongong, she joined the Faculty of Engineering in 2008. She is currently a senior professor of Faculty of Engineering \& Information Sciences. Her work focuses on practical applications of various nanomaterials as electrode materials for energy storage or conversion technologies, including rechargeable batteries, hydrogen storage, and supercapacitors. 
(a)
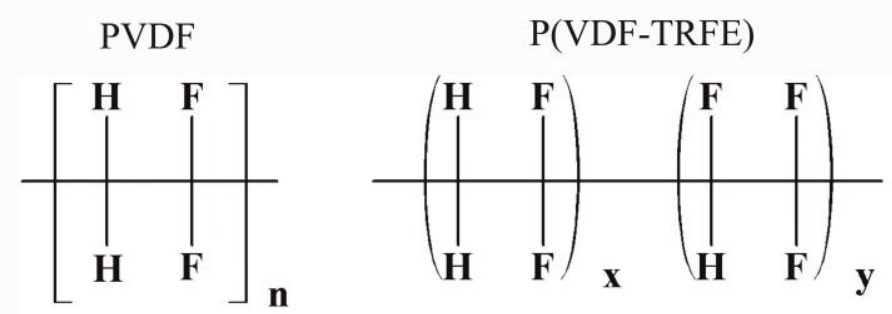

P(VDF-co-CTFE)

(b)

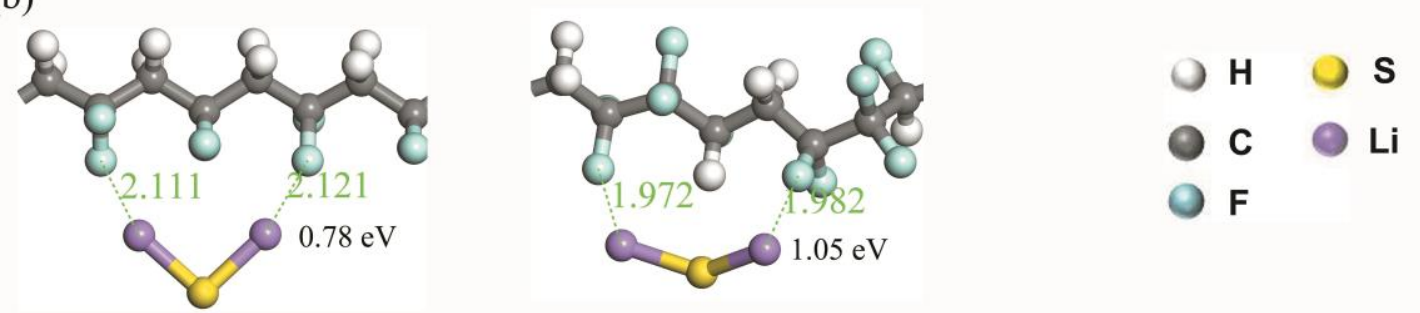

(c)
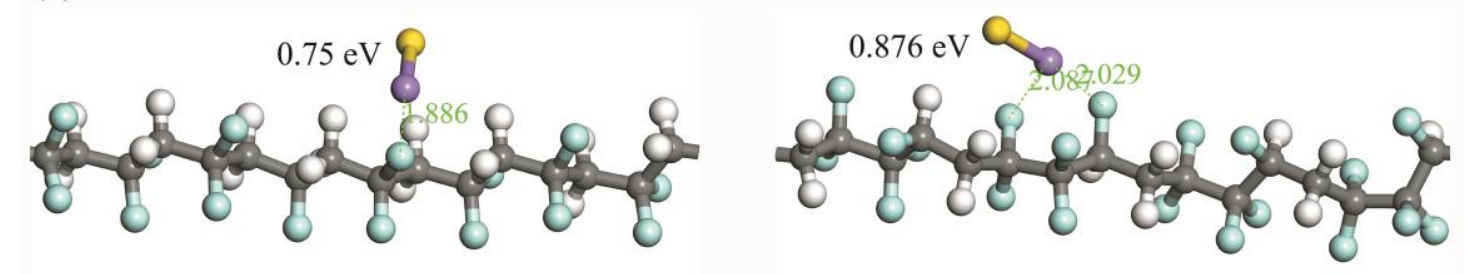

Figure 1. (a) Chemical structures of PVDF, P(VDF-TRFE), and P(VDF-co-CTFE) binders; $a b$ initio simulations showing the most stable binding configurations of (b) $\mathrm{Li}_{2} \mathrm{~S}$ and (c) Li-S· species with PVDF (left) and P(VDF-TRFE) (right) binders. 

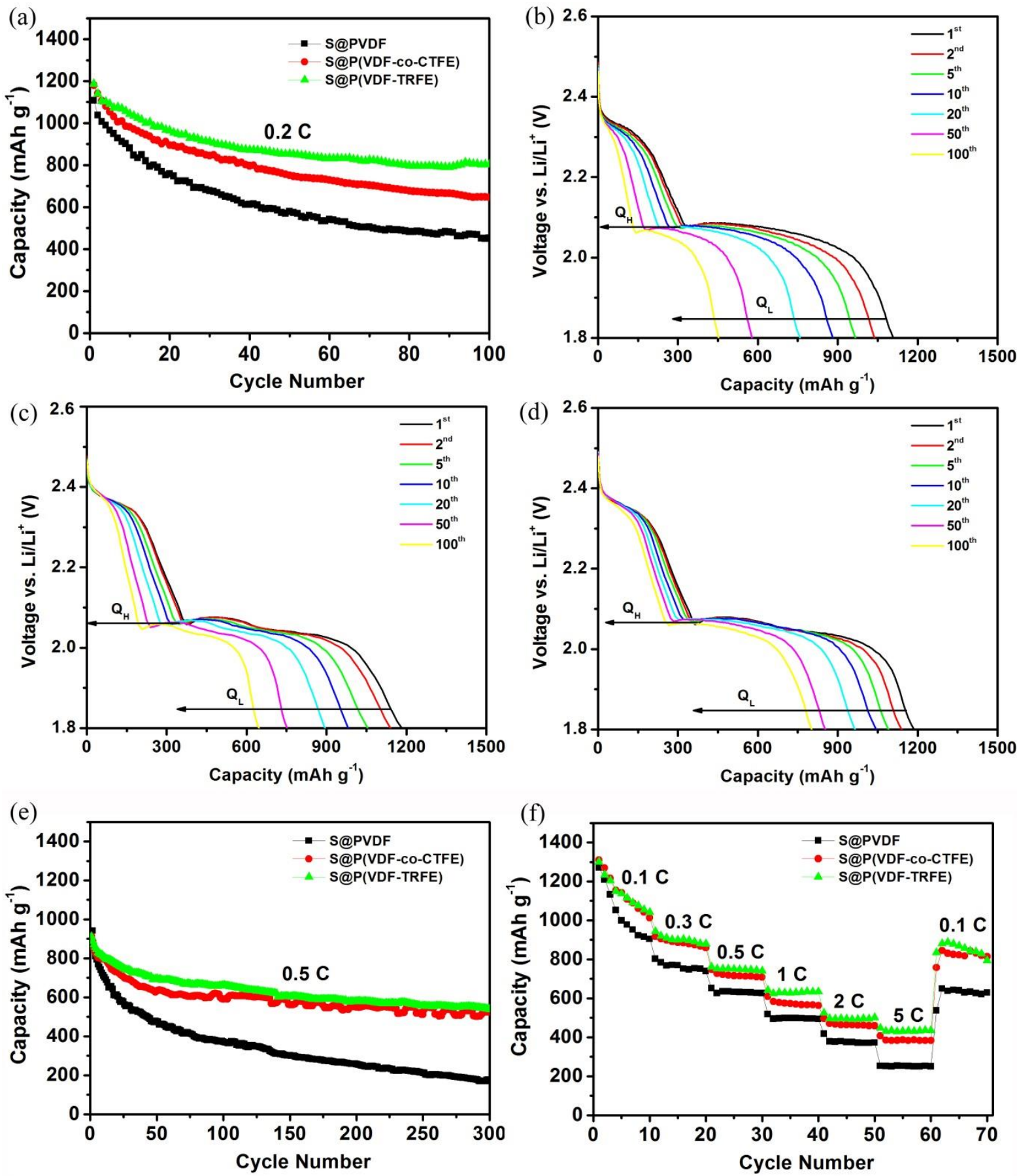

Figure 2. (a) Cycling stability of sulfur electrodes with different binders at current density of $0.2 \mathrm{C}$; galvanostatic charge-discharge profiles for selected cycles of (b) S@PVDF, (c) S@P(VDF-co-CTFE), and (d) S@P(VDF-TRFE) cathodes. (e) Long-term cycling performance at $0.5 \mathrm{C}$ and (f) rate capability of S@PVDF, S@P(VDF-co-CTFE), and S@P(VDF-TRFE) electrodes. 

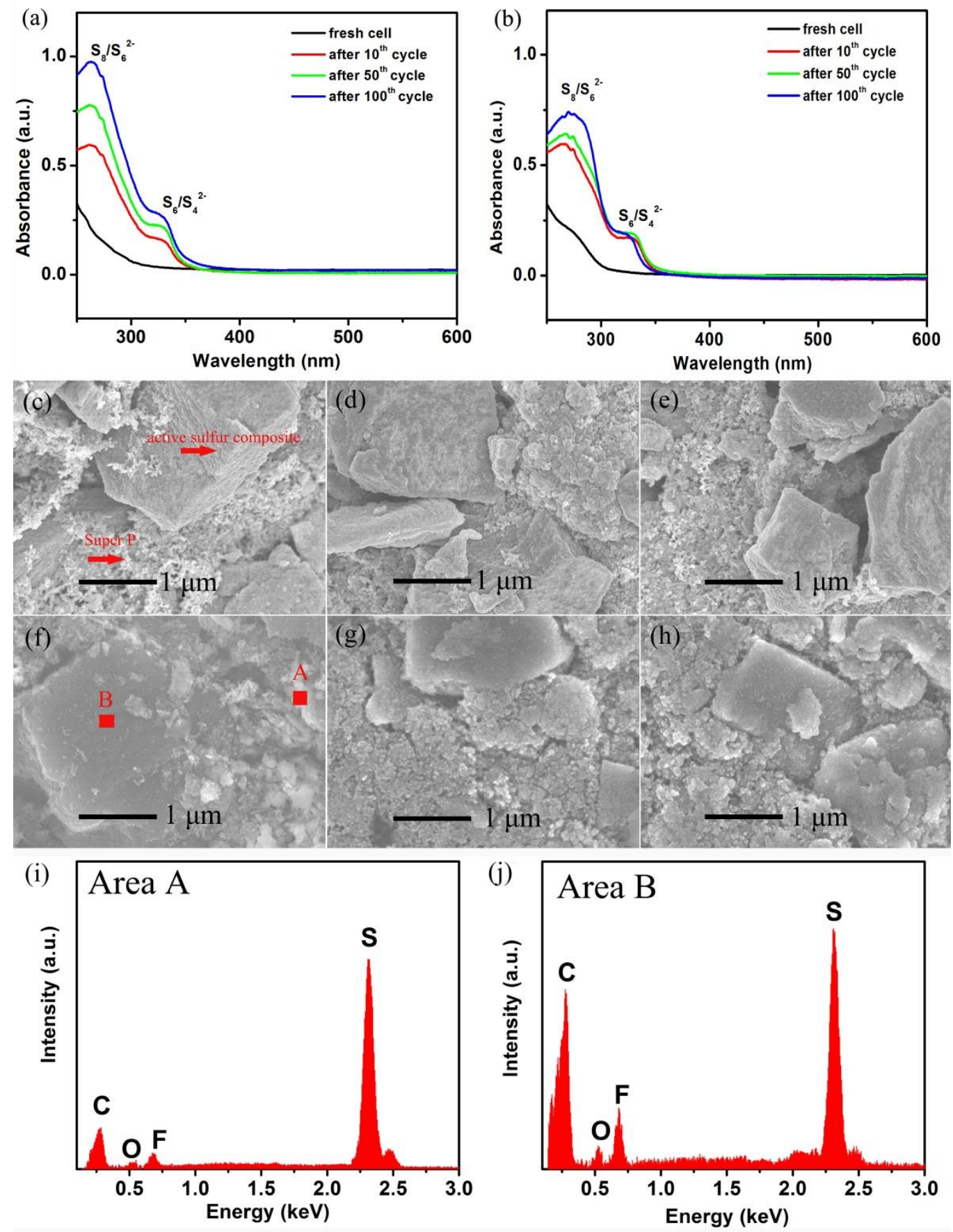

Figure 3. UV-vis absorption spectra of DOL/DME solution with (a) S@PVDF electrode and (b) S@P(VDF-TRFE) electrode for selected cycles; SEM images of fresh electrodes: (c) S@PVDF, (d) S@P(VDF-co-CTFE), and (e) S@P(VDF-TRFE), and of the electrodes after 50 cycles: (f) S@PVDF, (g)S@P(VDF-co-CTFE), and (h) S@P(VDF-TRFE) electrodes; (i, j) EDX spectra for selected areas of cycled S@PVDF electrode indicated in (f). 